\title{
Journal of Neurosurgery's Top 25 highly cited articles on Gamma Knife surgery for meningioma
}

\author{
Jason P. Sheehan, MD, PhD \\ Department of Neurological Surgery, University of Virginia Health System, Charlottesville, Virginia
}

KEY WORDS stereotactic radiosurgery, Gamma Knife, meningioma, citations

$\mathrm{O}$ ver the years, the Journal of Neurosurgery (JNS) has often published collections of works focused around a theme or topic. Such collections tend to benefit JNS's wide neurosurgical audience by providing an opportunity to revisit landmark studies. The collections also serve those who work in the designated field a chance to reflect on the changing landscape within a subspecialty area of neurosurgery.

The current collection is composed of 25 of the most highly cited articles on Gamma Knife radiosurgery for meningioma. The citations are measured based on an analysis from Web of Science. The publication dates of articles in this collection span the period from 1991 to 2015. Some of the earliest studies represent single-center studies with 50 patients, about whom the authors fairly and accurately reported favorable outcomes with relatively short follow-up intervals $\left(<3\right.$ years for most patients). ${ }^{1}$ While meaningful at the time, the field of Gamma Knife radiosurgery has certainly evolved to comprise higher levels of evidence. More recent articles represent international multicenter studies covering hundreds of meningioma patients with more than 10 years of follow-up. ${ }^{3}$ The field of Gamma Knife radiosurgery has matured into an era of big data, and the studies within it now benefit from cooperative research groups such as the International Gamma Knife Research Foundation (formerly the North American Gamma Knife Society) and the Japanese Leksell Gamma Knife Society. ${ }^{3}$ For patients with WHO Grade I meningiomas, clinical outcomes (such as neurological function, progression-free survival, radiation-induced complications, etc.) are measured over many years, and outcomes of a decade or more after the procedure are now routinely detailed in published articles. Gamma Knife radiosurgery has raised the bar in outcomes studies in the neurosurgical literature. Also, larger cohort sizes and outcomes derived from many centers, rather than a single one, provide enhanced statistical power and validity as well as generalizability to many Gamma Knife teams. Thus, the current studies provide a more accurate reflection of the benefit-to-risk profile of Gamma Knife radiosurgery for patients. Few areas of neurosurgery have undergone such a remarkable transition over a time span of 2 to 3 decades.

The trend in publications (Fig. 1) reflects an escalation in highly cited works from the 1990s through the 2000 s and on into the 2010s. While much has changed over the past 30 years, including paradigm shifts in neurosurgical subspecialties such as cerebrovascular disease, the time period over which the highly cited publications arise is a testament to the durability of Lars Leksell's concept of the Gamma Knife and his brain-dedicated radiosurgical device. $^{2}$ The escalation in meningioma publications also indicates a vibrancy in the field and the prominent role of Gamma Knife radiosurgery in the management of meningioma cases.

The topics covered by the highly cited articles in this collection furthermore demonstrate a breadth of applications for meningiomas (Fig. 2). Earlier articles tended to focus on the application of Gamma Knife radiosurgery to recurrent and residual meningiomas, but many now cover the use of Gamma Knife radiosurgery as an upfront treatment for patients harboring meningiomas. Also, the Gamma Knife is the most versatile of all neurosurgical 


\section{Number of Top Cited Articles}

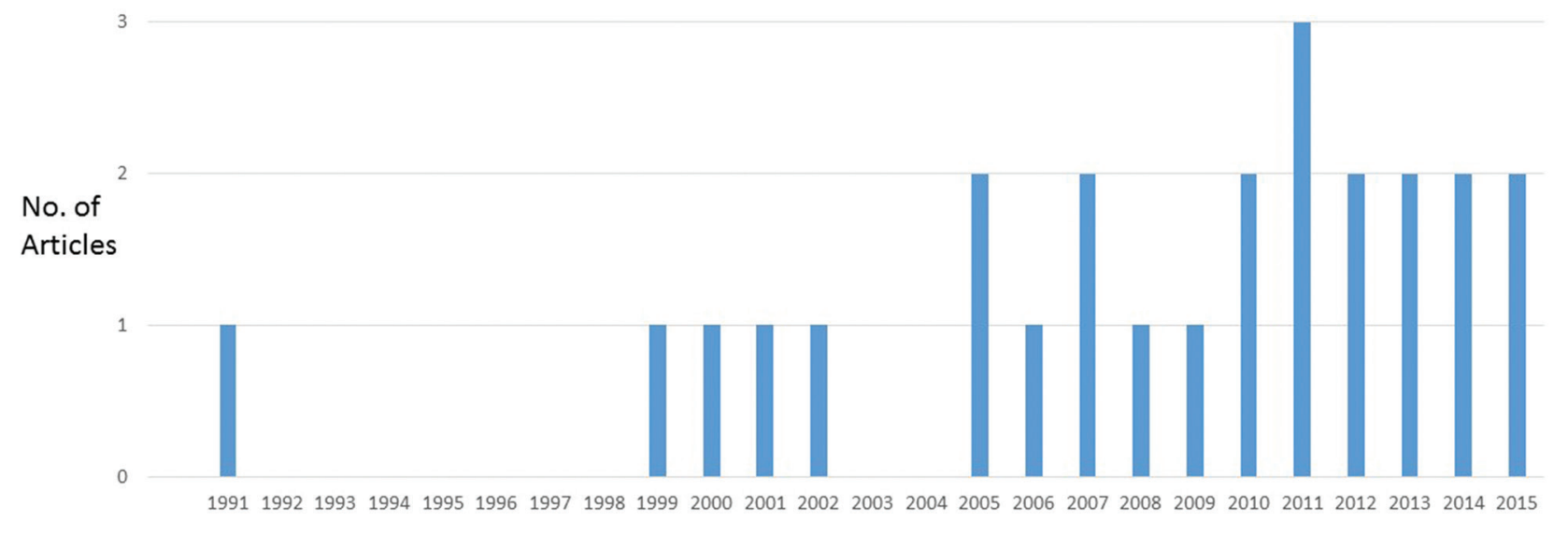

FIG. 1. Number of highly cited works per year of publication.

\section{Year of Publication of Article}

approaches for the treatment of meningiomas, because radiation can reasonably be delivered to meningiomas in virtually all intracranial locations. No other single neurosurgical approach has the range of efficacy and safety for variously situated meningiomas that the Gamma Knife has demonstrated in these articles.

The current collection of highly cited works provides us with the opportunity to reflect on past efforts in the field of Gamma Knife radiosurgery. It should also give neurosurgeons a sense of pride and satisfaction with the progress made in revolutionizing the treatment approach for patients harboring intracranial meningiomas. Neurosurgical treatment should never be worse for meningioma patients than the underlying disease. Gamma Knife radiosurgery has become a mainstay of treatment for patients with benign, atypical, and malignant meningiomas at the time of recurrence and in appropriately selected cases as the primary treatment. The current JNS supplement perhaps most importantly gives us a glimpse of the trends and advances to come in this field. There is no doubt that future refinements in Gamma Knife radiosurgery for meningiomas and myriad other indications will be chronicled in the JNS. With Gamma Knife radiosurgery for meningiomas, the best is yet to come.

\section{References}

1. Kondziolka D, Lunsford LD, Coffey RJ, Flickinger JC. Stereotactic radiosurgery of meningiomas. J Neurosurg 74:552-559, 1991

2. Leksell L. The stereotaxic method and radiosurgery of the brain. Acta Chir Scand 102:316-319, 1951

3. Sheehan JP, Starke RM, Kano H, Kaufmann AM, Mathieu D, Zeiler FA, et al. Gamma Knife radiosurgery for sellar and

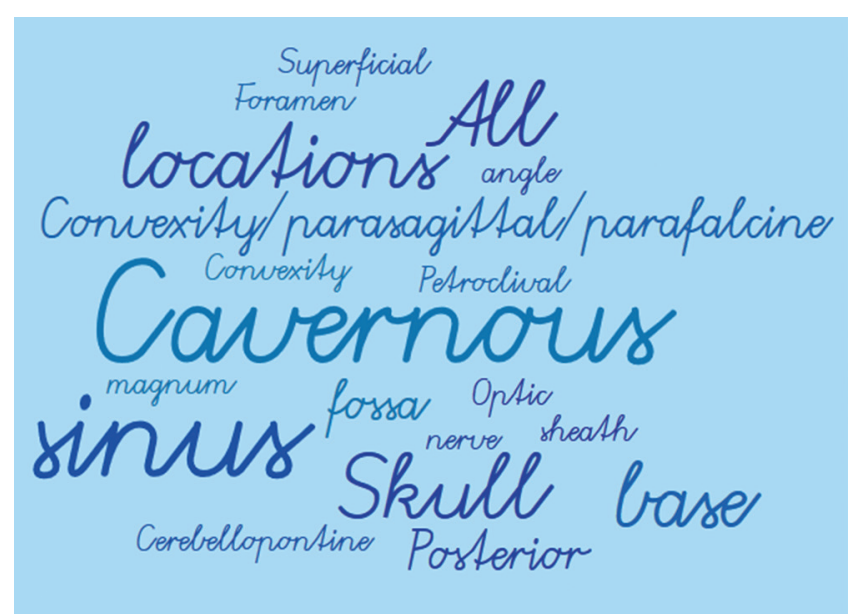

FIG. 2. Word cloud of meningioma articles based on the frequency of focus for the highly cited published works.

parasellar meningiomas: a multicenter study. J Neurosurg 120:1268-1277, 2014

\section{Disclosures}

The author reports no conflict of interest.

\section{Correspondence}

Jason Sheehan, Department of Neurological Surgery, University of Virginia Health System, P.O. Box 800212, Charlottesville, VA 22908. email jps2f@virginia.edu. 